\title{
COVID-19 and Hartnup disease: an affair of intestinal amino acid malabsorption
}

\author{
Enzo Nisoli ${ }^{1}$ (D) Saverio Cinti $^{2} \cdot$ Alessandra Valerio $^{3}$
}

Received: 13 June 2020 / Accepted: 10 July 2020 / Published online: 20 July 2020

(c) Springer Nature Switzerland AG 2020

\begin{abstract}
Since the outbreak of COVID-19, clinicians have tried every effort to fight the disease, and multiple drugs have been proposed. However, no proven effective therapies currently exist, and different clinical phenotypes complicate the situation. In clinical practice, many severe or critically ill COVID-19 patients developed gastrointestinal (GI) disturbances, including vomiting, diarrhoea, or abdominal pain, even in the absence of cough and dyspnea. Understanding the mechanism of GI disturbances is warranted for exploring better clinical care for COVID-19 patients. With evidence collected from clinical studies on COVID-19 and basic research on a rare genetic disease (i.e., Hartnup disorder), we put forward a novel hypothesis to elaborate an effective nutritional therapy. We hypothesize that SARS-CoV-2 spike protein, binding to intestinal angiotensinconverting enzyme 2, negatively regulates the absorption of neutral amino acids, and this could explain not only the GI, but also systemic disturbances in COVID-19. Amino acid supplements could be recommended.
\end{abstract}

Level of evidence No level of evidence: Hypothesis article.

Keywords Amino acids $\cdot$ ACE2 $\cdot \mathrm{B}^{0} \mathrm{AT} 1 \cdot \mathrm{COVID}-19 \cdot$ Gastrointestinal disturbances $\cdot$ Hartnup disease

\section{Introduction}

The recent outbreak of severe acute respiratory syndrome coronavirus 2 (SARS-CoV-2), and the resulting coronavirus disease 2019 (COVID-19) has had an enormous impact worldwide. By June 5, 2020, the number of confirmed cases had increased to over 7.2 million globally, and deaths surpassed 413,000. In clinical practice, the typical manifestations of COVID-19 are fever, cough, and dyspnea. Various gastrointestinal (GI) symptoms may also occur at disease onset. A recent meta-analysis found that 29 out of 35 studies reported GI symptoms (most commonly nausea, vomiting,

Enzo Nisoli

enzo.nisoli@unimi.it

Alessandra Valerio

alessandra.valerio@unibs.it

1 Department of Biomedical Technology and Translational Medicine, Center for Study and Research on Obesity, University of Milan, Milan 20129, Italy

2 Department of Experimental and Clinical Medicine, Marche Polytechnic University, Ancona, Italy

3 Department of Molecular and Translational Medicine, University of Brescia, Brescia 25123, Italy diarrhoea, or loss of appetite) in COVID-19 patients at diagnosis, with a pooled prevalence of $15 \%$ [1]. The pooled analysis did not show statistically significant relations between GI symptoms and clinical outcomes in terms of rates of death [1]. Notably, however, the subgroup analysis showed that: (1) patients with severe COVID-19 had higher rates of GI symptoms compared with those with non-severe disease, and (2) patients with GI involvement had a higher prevalence of severe COVID-19 course, with acute respiratory distress syndrome or complications [1]. Thus, understanding the mechanism of GI disturbance in SARS-CoV-2 infection is necessary to explore the optimal clinical care for COVID19 patients.

\section{Ileal absorptive enterocytes express the entry machinery used by SARS-CoV-2 to infect cells}

Angiotensin-converting enzyme 2 (ACE2) was identified as the receptor for SARS-CoV during the SARS epidemic in 2003 [2]. Virus entry requires viral spike (S) protein binding to ACE2 and priming by host cellular proteases. Recent reports show that SARS-CoV-2 engages ACE2 as 
entry receptor and employs the serine protease TMPRSS2 for $\mathrm{S}$ protein activation on target cells [3, 4]. In humans, ACE2 is expressed in airway epithelia, kidney cells, small intestine, lung parenchyma, vascular endothelia, as well as in neurons and glial cells [5]. A high number of ACE2 and TMPRSS 2 co-expressing cells reside in the small intestine, principally within the ileum, jejunum, and, to a lesser extent, the liver and colon; notably, ileal ACE2 ${ }^{+} \mathrm{TMPRSS}^{+}$cells are absorptive enterocytes [6].

ACE2 is a central component of the renin-angiotensin system. While its homologue ACE cleaves angiotensin I to generate angiotensin II (which drives pulmonary oedema and severe acute lung failure), ACE2 inactivates angiotensin II acting as a negative regulator of the system, thus exerting protective functions against lung pathology [7]. Upon binding of SARS-CoV, the ACE2 extracellular domain is cleaved, while its transmembrane domain is internalized and transferred into early endosomes. Notably, SARS-CoV infection or in vivo treatment with recombinant SARS-CoV $S$ protein downregulate ACE2 protein expression in mice lungs [8]. As a consequence, reduced ACE2 expression during SARS-CoV infection contributes to the pathogenesis of severe acute lung failure in SARS.

SARS-CoV-2 RNA has been detected in fecal specimens from pediatric and adult COVID-19 patients, persisting even after negative nasopharyngeal tests [9], leading to speculate that viral targeting of ileal ACE2 ${ }^{+}$TMPRSS2 $^{+}$enterocytes cells might be the cause of GI symptoms (Fig. 1). Further work is needed to clarify the effects of SARS-CoV-2 infection on enterocyte ACE2 expression and the possible consequences on local and systemic disease progression.

\section{ACE2, intestinal amino acid transport, and Hartnup disorder}

ACE2 is a chimeric protein displaying homology with ACE in the extracellular catalytic domain and with collectrin (Tmem27) in the membrane-anchoring domain [10]. Collectrin acts as a binding partner of the solute carrier family 6 (SLC6) amino acid transporter $\mathrm{B}^{0} \mathrm{AT} 1$ in kidney proximal tubule, regulating its trafficking on the luminal cell surface and its neutral amino acid transporter activity [11]. Collectrin is nearly absent in small intestine, the other primary site of $\mathrm{B}^{0} \mathrm{AT} 1$ expression. On the contrary, ACE2 does not bind to amino acid transporters in kidneys, but it is highly expressed in the intestine, where it acts as a specific binding partner of the luminal $\mathrm{B}^{0} \mathrm{AT} 1$ [12]. In ACE2 knockout mice, luminal expression of $\mathrm{B}^{0} \mathrm{AT} 1$ in the intestine is completely lost [12], demonstrating its fundamental role for the intestinal absorption of neutral amino acids (i.e., tryptophan, alanine, asparagine, glutamine, histidine, isoleucine, leucine, phenylalanine, serine, threonine, tyrosine, and valine) [12].
Interestingly, therapy with $\mathrm{ACE}$ inhibitors was found to increase intestinal expression of both ACE2 and $\mathrm{B}^{0} \mathrm{AT} 1$ [13].

Hartnup disorder is a rare inherited condition early described in 1956. Mutations in the SLC6A19 gene encoding $\mathrm{B}^{0} \mathrm{AT} 1$ have been identified as a cause of the disease, resulting in defective amino acid transport in the kidney and small intestine $[14,15]$. Besides the characteristic neutral aminoaciduria, patients may develop pellagra-like skin rash, variable neurologic manifestations (e.g., cerebellar ataxia, dysarthria, seizures, headache, dizziness), or psychiatric symptoms (e.g., anxiety, rapid mood changes, delirium), as well as diarrhoea. Symptoms may begin in childhood or early adulthood, mostly under stress conditions like malnutrition or infections, and seem mainly related to the reduced tryptophan absorption, which in turn causes improperly low production of nicotinamide (also known as niacin), necessary for the biosynthesis of nicotinamide adenine dinucleotide $\left(\mathrm{NAD}^{+}\right)$. Notably, the neurological and psychiatric symptoms of Hartnup patients evoke the most common neuropsychiatric symptoms of COVID-19 [5].

The mutations that interfere with ACE2 and $\mathrm{B}^{0} \mathrm{AT} 1$ interaction cause altered amino acid absorption. Of note, recent work has elucidated 47 potential functional missense variants from genomic databases within ACE2/SLC6A19/ TMPRSS2, warranting genomic enrichment analyses in SARS-CoV-2 patients, useful for the ongoing pandemic [16]. Experimental studies demonstrated that ACE2 knockout mice display highly increased susceptibility to intestinal inflammation and diarrhoea, which were reverted by dietary nicotinamide [17]. Altered composition of the gut microbiota (as a consequence of impaired amino acid transport and reduced secretion of antimicrobial peptides by Paneth cells in the small intestine) and changes in innate immunity contribute to the colitis phenotype observed in ACE2 knockout mice [17]. Together, these facts may explain diarrhoea and intestinal inflammation observed in Hartnup disorder, pellagra, or under conditions of severe malnutrition [17]. Similarly, diarrhoea and the other GI symptoms observed in COVID-19 can be explained by the SARS-CoV-2-sequestred ACE2 in intestinal absorptive enterocytes (Fig. 1). Notably, under chronic amino acid-supplemented diet, $\mathrm{B}^{0} \mathrm{AT} 1$ protein is increased in the proximal small intestine, while ACE2 protein levels raise in the distal one [18], suggesting that nutrition may play an important therapeutic role in both Hartnup and COVID-19 disorder.

\section{Hypothesis}

Based on clinical observations and basic research, we hypothesise that, in response to the SARS-CoV-2 binding to intestinal ACE2, the absorption of neutral amino acids is negatively regulated in COVID-19 patients. Upon 


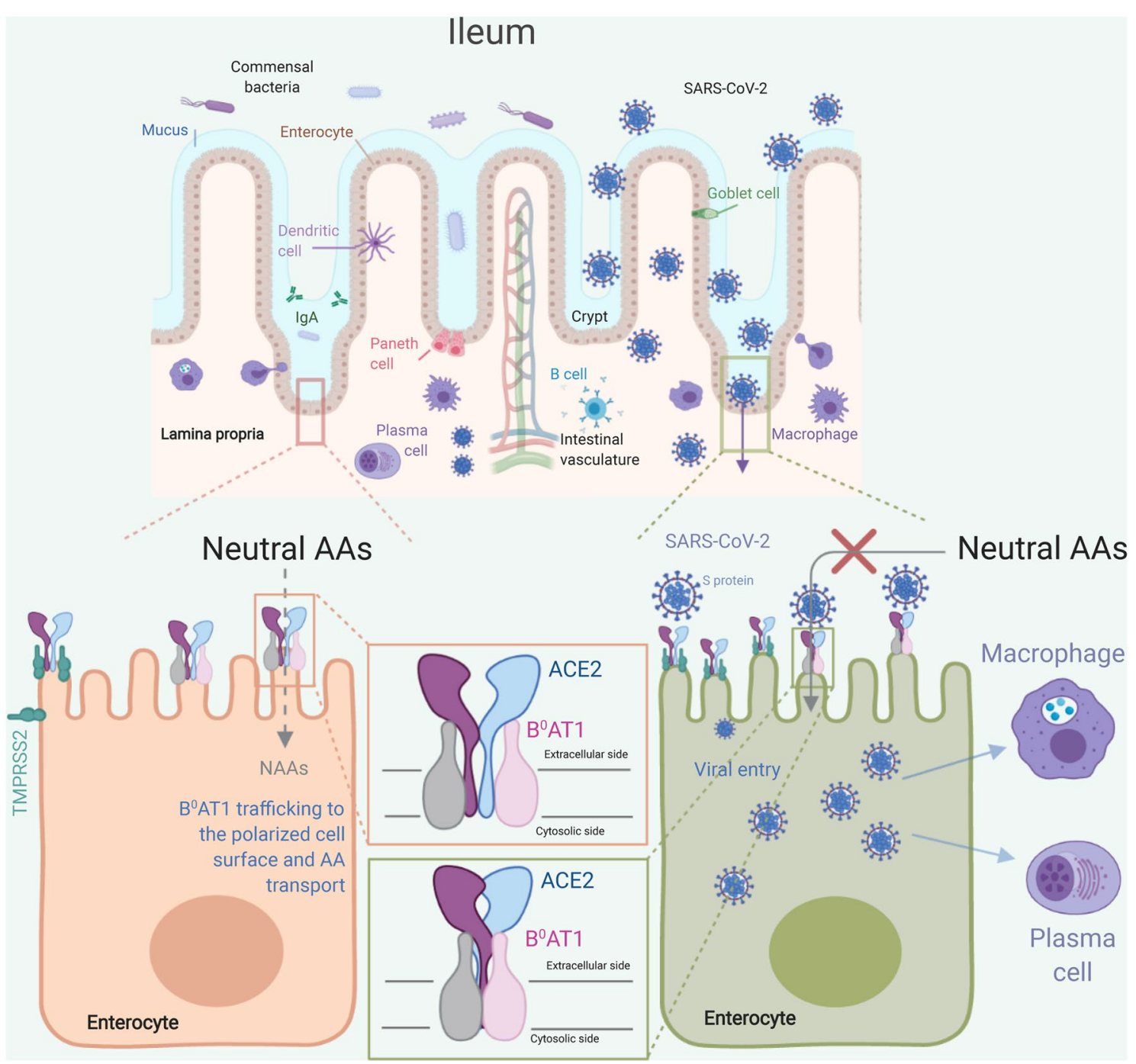

Fig. 1 SARS-CoV-2 binding to ACE2 in the small intestine, principally ileum and jejunum, may cause functional changes of enterocytes and start the innate immune response. If host defense mechanisms are defective, massive viral replication may occur, leading to hyperinflammation and severe systemic complications. ACE2 also functions as the chaperone for surface expression of the amino acid transporter $\mathrm{B}^{0} \mathrm{AT} 1$, which mediates the uptake of neutral amino acids into ileal enterocytes. Mutations in $\mathrm{B}^{0} \mathrm{AT} 1$ cause Hartnup disorder, an inherited defect of amino acid transport, whose symptoms are reminiscent of those of COVID-19. Cryo-electron microscopy structure

this change, neutral amino acids (and probably small proteins) accumulate in the intestinal lumen, promoting diarrhoea with protein malabsorption, microbiota changes, and immune defects. These pathological events may be causes of the multiple symptoms observed in COVID-19 patients, both in early and late phases of the disease. Immune response to SARS-CoV-2 was suggested-especially in pulmonary parenchyma-to be markedly affected by inflammation processes started by resident macrophages or epithelial cells and followed by the production of proinflammatory of the full-length human ACE2-B ${ }^{0} \mathrm{AT} 1$ complex has been recently described [23]. The ACE2 forms homodimers which are sandwiched by $\mathrm{B}^{0} \mathrm{AT} 1$, resulting in $\mathrm{ACE} 2-\mathrm{B}^{0} \mathrm{AT} 1$ complex. Structural analysis suggests that two SARS-CoV-2 spike (S) protein trimers simultaneously bind to an ACE2 homodimer. While the ACE2- $\mathrm{B}^{0} \mathrm{AT} 1$ complex exists in the open or closed state, the SARS-CoV-2-ACE2-B ${ }^{0}$ AT1 ternary complex only displays the closed conformation. $A A$ amino acids. This figure was created by an author (E.N.) using the website https:// app.biorender.com

cytokines and chemokines and recruitment of circulating monocytes. Further, the reduction and dysfunction of lymphocytes impedes the effective onset of the adaptive immune response. Uncontrolled virus infection leads to a vicious cycle and further worsening of lung injury and multiorgan dysfunction [19]. Mounting the innate and adaptive immune response is a highly energy-consuming process. To timely undertake the required cellular tasks, immune cells undergo a dramatic reprogramming of their cellular metabolism, which enables the production of ATP and the synthesis 
of new biomolecules. Thus, the flow of nutrient substrates (glucose, amino acids, and fatty acids) is redirected towards immunity and away from storage or other non-immune organismal processes. In malnourished patients or conditions of intestinal amino acid malabsorption, as in the COVID-19 or Hartnup patients, the adaptive immune response cannot be effectively initiated because the absorption of essential energy substrates is impaired by SARS-CoV-2 binding to ACE2. Meanwhile, the diversion of energy substrates to the maintenance of the immune system, in addition to reduced $\mathrm{NAD}^{+}$synthesis, causes insufficient protein synthesis and energy production in skeletal muscle and brain. Similarly, the reduced serotonin synthesis may affect brain function and explain some neurological and psychiatric symptoms in COVID-19.

A high-protein diet can overcome the deficient transport of neutral amino acids in most Hartnup patients. Thus, we propose to treat COVID-19 patients, both acutely (in the early phase of the disease) and chronically (at least for subsequent 3-6 months after dismissal), with specific amino acid supplements and niacin. Very recently, we and others have proved the ability of designer amino acid-supplemented diets to promote energy production, amino acid absorption, protein synthesis, healthy gut microbiota, and immune response $[20,21]$. This approach may be crucial to prevent long-term physical, neurologic, and psychiatric sequelae in COVID-19 patients after recovery.

Future research is needed to explore whether SARS$\mathrm{CoV}-2$ directly attacks absorptive enterocytes-as suggested recently [22], and to examine the effect of SARS-CoV-2 on intestinal and renal absorption of neutral amino acids. Efforts are also necessary to confirm whether and how SARS-CoV-2 influences the intestinal adaptive immune response. Preclinical and clinical studies should investigate the impact of amino acid supplements, with or without niacin, on the outcome of SARS-CoV-2 infection. Based on the ability to increase intestinal expression of both ACE2 and $\mathrm{B}^{0} \mathrm{AT} 1$ [13], ACE inhibitors could potentiate the efficacy of the dietary supplements. Randomised clinical trials are mandatory to assess the effectiveness of nutritional therapies on physical and psychological resilience in COVID-19 patients.

\section{What is already known on this subject?}

Many severe COVID-19 patients developed gastrointestinal (GI) disturbances, even in the absence of cough and dyspnea. Understanding the mechanism of GI disturbances is warranted for exploring better clinical care for COVID-19 patients. The Hartnup disease is a rare inherited disease, in which the ACE2-dependent trafficking of $\mathrm{B}^{0} \mathrm{AT} 1-\mathrm{a}$ transporter of neutral amino acids-is genetically defective. Notably, Hartnup disease includes cutaneous alterations, neurologic manifestations, or psychiatric symptoms, frequently described in severe COVID-19 cases, together with the GI disturbances. Thus, we hypothesise that COVID19 may share a pathophysiological mechanism with the Hartnup disease.

\section{What does this study add?}

As a consequence of our hypothesis, we propose to treat COVID-19 patients as Hartnup patients. High-protein diets indeed have been found to overcome the deficient transport of neutral amino acids in most Hartnup patients. This approach may be crucial to prevent long-term physical, neurologic, and psychiatric sequelae in COVID-19 patients after recovery.

Acknowledgements The research work in the laboratories of E.N. and A.V. is partly supported by Cariplo Foundation (Grant 1006-2016) and Professional Dietetics (Milan, Italy).

Author contributions EN and AV did the literature search, put forward the hypothesis, and drafted the manuscript. EN, SC and AV revised the manuscript.

\section{Compliance with ethical standards}

Conflict of interest The authors declare that they have no conflict of interest.

Ethical approval This article does not contain any studies with human participants performed by any of the authors.

Informed consent For this type of study (Hypothesis study) formal consent is not required.

\section{References}

1. Mao R, Qiu Y, He JS et al (2020) Manifestations and prognosis of gastrointestinal and liver involvement in patients with COVID19: a systematic review and meta-analysis. Lancet Gastroenterol Hepatol. https://doi.org/10.1016/S2468-1253(20)30126-6

2. Li W, Moore MJ, Vasllieva N et al (2003) Angiotensin-converting enzyme 2 is a functional receptor for the SARS coronavirus. Nature 426:450-454. https://doi.org/10.1038/nature02145

3. Hoffmann M, Kleine-Weber H, Schroeder S et al (2020) SARSCoV-2 cell entry depends on ACE2 and TMPRSS2 and is blocked by a clinically proven protease inhibitor. Cell 181:271-280.e8. https://doi.org/10.1016/j.cell.2020.02.052

4. Walls AC, Park YJ, Tortorici MA et al (2020) Structure, function, and antigenicity of the SARS-CoV-2 spike glycoprotein. Cell 181:281-292.e6. https://doi.org/10.1016/j.cell.2020.02.058

5. Zubair AS, McAlpine LS, Gardin T et al (2020) Neuropathogenesis and neurologic manifestations of the coronaviruses in the age of coronavirus disease 2019: a review. JAMA Neurol. https://doi. org/10.1001/jamaneurol.2020.2065

6. Ziegler CGK, Allon SJ, Nyquist SK et al (2020) SARS-CoV-2 receptor ACE2 is an interferon-stimulated gene in human airway epithelial cells and is detected in specific cell subsets across tissues. Cell 181:1016-1035. https://doi.org/10.1016/j. cell.2020.04.035 
7. Imai Y, Kuba K, Rao S et al (2005) Angiotensin-converting enzyme 2 protects from severe acute lung failure. Nature 436:112116. https://doi.org/10.1038/nature03712

8. Kuba K, Imai Y, Rao S et al (2005) A crucial role of angiotensin converting enzyme 2 (ACE2) in SARS coronavirus-induced lung injury. Nat Med 11:875-879. https://doi.org/10.1038/nm1267

9. Xu Y, Li X, Zhu B et al (2020) Characteristics of pediatric SARSCoV-2 infection and potential evidence for persistent fecal viral shedding. Nat Med 26:502-505. https://doi.org/10.1038/s4159 1-020-0817-4

10. Zhang H, Wada J, Hida K et al (2001) Collectrin, a collecting duct-specific transmembrane glycoprotein, is a novel homolog of ACE2 and is developmentally regulated in embryonic kidneys. J Biol Chem 276:17132-17139. https://doi.org/10.1074/jbc.M0067 23200

11. Danilczyk U, Sarao R, Remy C et al (2006) Essential role for collectrin in renal amino acid transport. Nature 444:1088-1091. https://doi.org/10.1038/nature05475

12. Camargo SMR, Singer D, Makrides V et al (2009) Tissue-specific amino acid transporter partners ACE2 and collectrin differentially interact with hartnup mutations. Gastroenterology 136:872-882. https://doi.org/10.1053/j.gastro.2008.10.055

13. Vuille-Dit-Bille RN, Camargo SM, Emmenegger L et al (2015) Human intestine luminal ACE2 and amino acid transporter expression increased by ACE-inhibitors. Amino Acids 47:693705. https://doi.org/10.1007/s00726-014-1889-6

14. Kleta R, Romeo E, Ristic Z et al (2004) Mutations in SLC6A19, encoding B0AT1, cause Hartnup disorder. Nat Genet 36:9991002. https://doi.org/10.1038/ng1405

15. Seow HF, Bröer S, Bröer A et al (2004) Hartnup disorder is caused by mutations in the gene encoding the neutral amino acid transporter SLC6A19. Nat Genet 36:1003-1007. https://doi. org/10.1038/ng1406

16. Gupta R, Charron J, Stenger CL et al (2020) SARS-CoV-2 (COVID-19) structural and evolutionary dynamicome: insights into functional evolution and human genomics. J Biol Chem. https ://doi.org/10.1074/jbc.ra120.014873 (Online ahead of print)

17. Perlot T, Penninger JM (2013) ACE2-from the renin-angiotensin system to gut microbiota and malnutrition. Microbes Infect $15: 866-873$

18. Jando J, Camargo SMR, Herzog B, Verrey F (2017) Expression and regulation of the neutral amino acid transporter B0AT1 in rat small intestine. PLoS One. https://doi.org/10.1371/journ al.pone. 0184845

19. Li H, Liu L, Zhang D et al (2020) SARS-CoV-2 and viral sepsis: observations and hypotheses. Lancet 395:1517-1520

20. D'Antona G, Ragni M, Cardile A et al (2010) Branched-chain amino acid supplementation promotes survival and supports cardiac and skeletal muscle mitochondrial biogenesis in middleaged mice. Cell Metab 12:362-372. https://doi.org/10.1016/j. cmet.2010.08.016

21. Yang Z, Huang S, Zou D et al (2016) Metabolic shifts and structural changes in the gut microbiota upon branched-chain amino acid supplementation in middle-aged mice. Amino Acids 48:2731-2745. https://doi.org/10.1007/s00726-016-2308-y

22. Lamers MM, Beumer J, van der Vaart J et al (2020) SARS-CoV-2 productively infects human gut enterocytes. Science. https://doi. org/10.1126/science.abc1669 (Online ahead of print)

23. Yan R, Zhang Y, Li Y, Xia L, Guo Y, Zhou Q (2020) Structural basis for the recognition of SARS-CoV-2 by full-length human ACE2. Science 367:1444-1448

Publisher's Note Springer Nature remains neutral with regard to jurisdictional claims in published maps and institutional affiliations. 